\title{
A Fast Algorithm for Planning Optimal Platoon Speeds on Highway
}

\author{
Qichen Deng* Xiaoliang Ma* \\ * ITS Lab, Traffic and Logistics, Royal Institute of Technology (KTH), \\ Teknikringen 72, SE-10044, Sweden, \\ (Corresponding email: liang@kth.se)
}

\begin{abstract}
To meet policy requirements on increased transport energy efficiency and reduced emissions, smart control and management of vehicles and fleets have become important for the development of eco-friendly intelligent transportation systems (ITS). The emergence of new information and communication technologies and their applications, particularly vehicleto-vehicle and vehicle-to-infrastructure communication, facilitates the implementation of autonomous vehicle concepts, and meanwhile serves as an effective means for control of vehicle fleet by continuously providing support and guidance to drivers. While convoy driving of trucks by longitudinal automation could save $5-15 \%$ of fuel consumption due to the reduction of airdrag resistance, this study attempts to investigate the energy saving potential of truck platoons by intelligent speed planning. Assuming that real-time traffic information is available because of communication, an efficient speed control algorithm is proposed based on optimal control theory. The method is faster than the conventional dynamic programming approach and hence applied in the study to analyze energy saving potential of simple platoon operations including acceleration and deceleration. The numerical result shows significant improvement on energy saving due to speed planning during platooning. It can be further applied for more complex platooning operations.
\end{abstract}

Keywords: Platooning operations, speed planning, acceleration and deceleration, adaptive cruise control, optimization.

\section{INTRODUCTION}

The rapid growth in economy is coupled with increasing transport demand, energy consumption and greenhouse gas emission. The entire transport system is nowadays facing great challenges due to the fact that the demand is growing steadily year by year while the negative impact on the environment needs to be minimized and the requirement on sustainability of traffic system has to be fulfilled. According to statistics, transport in the European Union corresponds to $25 \%$ of greenhouse gases and $30 \%$ of $\mathrm{CO}_{2}$. Freight transport by heavy-duty vehicles (HDV) has been considered as one of the main policy areas for overall energy efficiency improvement. On the other hand, modern advanced information and communication technology (ICT) provides opportunities to develop potential solutions to all these challenges.

\subsection{Background}

Platooning or grouping vehicles into platoons is a method of increasing the capacity of roads and reducing energy consumption [e.g. Shladover et al. 1991, Franke et al. 1995]. This has become a truck fleet management strategy, in which the leading vehicle is in free driving while the others automatically follow vehicle at the immediate front at short distance using adaptive cruise control (ACC). Recently, it is an active research area, and especially has a good chance becoming the first application in the wave of autonomous driving in the World. Excessive study has been carried out on vehicle and platoon control, while the benefits of platooning of heavy vehicles can be implemented with relatively low cost. In many studies including field trials, the average benefit can be up to $20 \%$ in fuel saving for highway driving due to less aerodynamic drag [Browand et al. 2004].

Several previous studies have investigated optimal control of single vehicle and platoon. For example, Hooker [1988] derived optimal control for single vehicle using dynamic programming approach. Godbole and Lygeros [1993] designed a longitudinal control to the leading car of a platoon so that the platoon can move on an automated highway with safe spacing and maintain corresponding optimal velocity. Stankovic et al. [2013] designed suboptimal longitudinal control using stochastic inclusion principle for platooning vehicles. It is however the strong interest from industry such as the international truck maker, Scania that promotes the idea to real demonstration and future application.

\subsection{Research Objectives}

This study is a part of the iQFleet project that is jointly initialized by Scania AB and KTH. The main objective of the project is to develop real-time fleet management systems for heavy-duty vehicles (HDV) implementing platooning. One part of the project extends the previous research in HDV controls (such as adaptive cruise control 
(ACC) and active braking systems (ABS)) based on onboard sensors (e.g. radar and lidar). Due to the cooperation with neighboring vehicles and road-side units, both individual vehicle and fleet controls can be designed in smarter ways.

Another part of the project focuses on predicting traffic information using smart sensing data such as GPS, cameras etc. Predicted traffic information provide good opportunities to plan platoon speed ahead at a tactical level. Previous study shows that vehicle could achieve more fuel saving and emission reduction by taking advantages of local congestion prediction [Ma 2013]. However, the dynamic programming (DP) based method tries to find global optimal solution in a discretized speed range, which is computationally expensive, not efficient enough for realtime application.

This paper continues exploring speed planning for a whole platoon. It attempts to derive optimal velocity trajectory for the leading vehicle of a platoon with respect to the objective of minimum energy consumption. This study tries to apply another Optimal Speed Planning (OSP) algorithm, to derive optimal speed profile of the leading vehicle, then retrieve the states of following vehicles in the platoon automated by ACC control. While the methodology has been applied for solving individual vehicle control problem, the contribution of the study is still unique due to the extension to the context of truck platooning.

The next section of the paper focuses on derivation of a speed planning algorithm for a single vehicle based on the Pontryagin Minimum Principle (PMP). The approach is then extended to a platoon of two trucks running on highway while taking into account air drag resistance. Numerical examples are demonstrated for the different cases.

\section{METHODOLOGY}

This section first formulates the mathematical problem in optimal control, and then derive the algorithm to find optimal trajectory of the platoon leader (in a two-vehicle platoon) with initial and ending state given to the leading vehicle. The section starts with notations and definitions, assuming that a platoon of two heavy trucks running on the highway with the same initial velocity.

\subsection{Notations}

$N$ - number for discretization in space

$i$ - index of discretization

$v_{i}$ — velocity of the leading vehicle

$\tilde{v}_{i}$ - velocity of the following vehicle

$v_{0}$ — initial velocity of the leading vehicle

$v_{f}$ - final velocity of the leading vehicle

$m$ - number for discretization in velocity

$s_{i}$ - position of the leading vehicle

$\tilde{s}_{i}$ - position of the following vehicle

$L$ - travel distance of the leading vehicle

$s_{h w}$ - desired headway between two vehicles $t_{i}$ - time instance at position $s_{i}$

$\Delta t_{i}$ - travel time from $s_{i}$ to $s_{i+1}$

$\Delta t_{i}=t_{i+1}-t_{i}$

$a_{i}$ - acceleration of the leading vehicle

$a_{a c c}$ - adaptive cruise control that used by the follower

$a_{\max }$ - maximum acceleration

$a_{\text {min }}-$ minimum acceleration (maximum deceleration)

$r(v, a)$ - energy consumption per second of leader $r(\tilde{v}, \tilde{a})$ - energy consumption per second of follower $r(v, a)$ is the energy consumption function which is proposed by Oguchi.

$v_{\min }=\min \left\{v_{0}, v_{f}\right\}$

$v_{\max }=\max \left\{v_{0}, v_{f}\right\}$

$\Delta v=\left(v_{\max }-v_{\min }\right) / m$

$v^{(k)}=v_{\min }+(k-1) \Delta v$

$\delta v_{i}=v_{i}-\tilde{v}_{i}$

$\Delta s=L / N$

$\delta s_{i}=s_{i}-\tilde{s}_{i}$

$c_{1}$ - safe distance between two vehicles

$c_{2}$ - time headway

$s_{h w, i}=c_{1}+c_{2} \tilde{v}_{i}$ where $c_{1}=5$ and $c_{2}=0.1$

\subsection{Adaptive Cruise Control}

In order to analyze the performance of platoon automation, this paper applies a relatively simple ACC controller presented by Donikian et al. [1998]:

$$
a_{a c c, i}^{*}=k_{1} \delta v_{i}+k_{2}\left(\delta s_{i}-c_{1}-\tilde{v}_{i} t_{0}\right)
$$

where the control parameters are $k_{1}=3 k_{2}=0.2$ and $t_{0} \in[0.1,0.25]$ is the time headway. The ACC control is further tuned to ensure the safe distance $\left(c_{1}=5\right)$ between two vehicles. The maximum distance that a follower can travel within time interval $\Delta t_{i}$ is given by:

$$
\Delta \tilde{s}_{\max , i}=s_{i+1}-\tilde{s}_{i}-c_{1}
$$

similarly, the minimum travel distance is:

$$
\Delta \tilde{s}_{\min , i}=s_{i+1}-\tilde{s}_{i}-s_{h w, i}
$$

where

$$
s_{i+1}=s_{i}+\Delta s
$$

the maximum ACC could be calculated from:

$$
a_{a c c, \max , i}=\frac{2 \Delta \tilde{s}_{\max }-2 \tilde{v}_{i} \Delta t_{i}}{\Delta t_{i}^{2}}
$$

so is the minimum ACC from:

$$
a_{a c c, \min , i}=\frac{2 \Delta \tilde{s}_{\min }-2 \tilde{v}_{i} \Delta t_{i}}{\Delta t_{i}^{2}}
$$

Moreover, the following constraints are applied on acceleration: 
Table 1. Oguchi's Energy Consumption Model

\begin{tabular}{|l|l|l|}
\hline \multirow{5}{*}{ Vehicle Parameters } & $Q$ & Total Energy Consumption \\
& $\mu$ & Coefficient of Friction \\
& $M$ & Coefficient of Air-drag \\
& $m$ & Mass \\
& $c_{D}$ & Internal Resistance Equivalent \\
& $A_{a}$ & Air Drag Coefficient \\
& $\rho_{a}$ & Front Area \\
& $\phi$ & Air Density \\
& $E_{(i d l e)}$ & Reduction Factor \\
\hline \multirow{5}{*}{ Driving Parameters } & $T$ & Travel Time \\
& $v$ & Vehicle Speed \\
\hline Physical Parameters & $g$ & Road Gradient \\
\hline
\end{tabular}

$a_{a c c, \max , i}=\left\{\begin{array}{cl}\frac{2 \Delta \tilde{s}_{\max }-2 \tilde{v}_{i} \Delta t_{i}}{\Delta t_{i}^{2}} & \text { if } a_{\min \leq} a_{a c c, \max , i} \leq a_{\max } \\ a_{\max } & \text { otherwise }\end{array}\right.$

$a_{a c c, \min , i}=\left\{\begin{array}{cl}\frac{2 \Delta \tilde{s}_{\min }-2 \tilde{v}_{i} \Delta t_{i}}{\Delta t_{i}^{2}} & \text { if } a_{\min \leq} a_{a c c, \min , i} \leq a_{\max } \\ a_{\min } & \text { otherwise }\end{array}\right.$

Hence, the actual ACC is decided by the following:

$$
a_{a c c, i}=\left\{\begin{array}{cc}
a_{a c c, \min , i} & \text { if } a_{a c c}^{*}<a_{a c c, \min , i} \\
a_{a c c, \max , i} & \text { if } a_{a c c}^{*}>a_{a c c, \max , i} \\
a_{a c c, i}^{*} & \text { otherwise }
\end{array}\right.
$$

\subsection{Problem Formulation}

The main idea in the study is to find the optimal speed trajectory of the leading truck of the platoon and then calculate the states of the other trucks by the ACC control. Similar to the previous dynamic programming approach for solving speed planning of a single vehicle [Ma 2013], discretization on space $\left(\Delta s=\frac{L}{N}\right)$ is applied for convenience. The general problem is therefore represented by the following optimization formulation

$$
\min \sum_{i=0}^{N-1} r\left(v_{i}, a_{i}\right) \Delta t_{i}
$$

s.t.

$$
\left\{\begin{array}{l}
s_{i+1}=s_{i}+\Delta s \\
\Delta s=v_{i} \Delta t_{i}+a_{i} \Delta t_{i}^{2} / 2 \\
v_{i+1}=v_{i}+a_{i} \Delta t_{i} \\
a_{i} \in\left[a_{\text {min }}, a_{\text {max }}\right] \\
v_{0}=v_{0} \\
v_{N}=v_{f} \\
s_{0}=0 \\
s_{N}=L
\end{array}\right.
$$

For the rest of the vehicles in a HDV platoon, given initial state $\tilde{v}_{0}$ and $\tilde{s}_{0}$, their intermediate and final states can be retrieved according to platoon leader's state (i.e., $v_{i}, a_{i}$ and $s_{i}$ ) by adaptive cruise control (ACC).

\subsection{Objective Function}

In this study, a theoretical fuel consumption model proposed by Oguchi et al. [2002] is applied i.e.

$$
Q=E_{\text {idle }}+E_{\text {eng }}
$$

where the first part is basic vehicle fuel consumption during idling phase

$$
\begin{gathered}
E_{i d l e}=\sum_{i=0}^{N-1} \int_{t_{i}}^{t_{i+1}} E_{i d l e, i} d t=\sum_{i=0}^{N-1} E_{i d l e, i} \Delta t_{i} \\
\Delta t_{i}=t_{i+1}-t_{i}
\end{gathered}
$$

The running fuel consumption is calculated by

$$
\begin{aligned}
& E_{\text {eng }}=\sum_{i=0}^{N-1} \delta_{i} E_{\text {eng }, i} \\
&=\sum_{i=0}^{N-1} \delta_{i}\left[\mu M g \cos \Theta \Delta s+\sin \Theta M g \int_{t_{i}}^{t_{i+1}} v d t\right. \\
&\left.+\kappa \int_{t_{i}}^{t_{i+1}} v^{3} d t+(M+m)\left(\frac{1}{2} v_{i+1}^{2}-\frac{1}{2} v_{i}^{2}\right)\right] \\
& v(t)=v_{0}+a(t) t .
\end{aligned}
$$

By assuming that interval acceleration

$$
a_{i}=\frac{v_{i+1}-v_{i}}{\Delta t_{i}}
$$

is constant in time interval $\left[t_{i}, t_{i+1}\right]$, we derive

$$
\begin{aligned}
\kappa \int_{t_{i}}^{t_{i+1}} v^{3} d t & =\kappa \int_{0}^{\Delta t_{i}}\left(v_{i}+a_{i} t\right)^{3} d t \\
& =\kappa\left[v_{i}^{3} \Delta t_{i}+\frac{3}{2} v_{i}^{2} a_{i} \Delta t_{i}^{2}+v_{i} a_{i}^{2} \Delta t_{i}^{3}+\frac{1}{4} a_{i}^{3} \Delta t_{i}^{4}\right] .
\end{aligned}
$$

To simplify the problem we assume $\Theta=0$ (road gradient 0 ) and $m=0$ (no internal resistance). This leads to

$$
\begin{aligned}
E_{\text {eng }, i} & =\mu M g \Delta s+\kappa\left(v_{i}^{3} \Delta t_{i}+\frac{3}{2} v_{i}^{2} a_{i} \Delta t_{i}^{2}\right. \\
& \left.+v_{i} a_{i}^{2} \Delta t_{i}^{3}+\frac{1}{4} a_{i}^{3} \Delta t_{i}^{4}\right)+\frac{1}{2} M\left(v_{i+1}^{2}-v_{i}^{2}\right)
\end{aligned}
$$

where $\kappa$ is given by:

$$
\kappa=\frac{1}{2} \rho_{a} A_{a} c_{D}(1-\phi)
$$

therefore the vehicle fuel consumption can be derived as

$$
\begin{aligned}
Q & =\sum_{i=0}^{N-1} r\left(v_{i}, a_{i}\right) \Delta t_{i} \\
& =\sum_{i=0}^{N-1}\left(E_{\text {idle }, i} \Delta t_{i}+\delta_{i} E_{\text {eng }, i}\right)
\end{aligned}
$$

where 


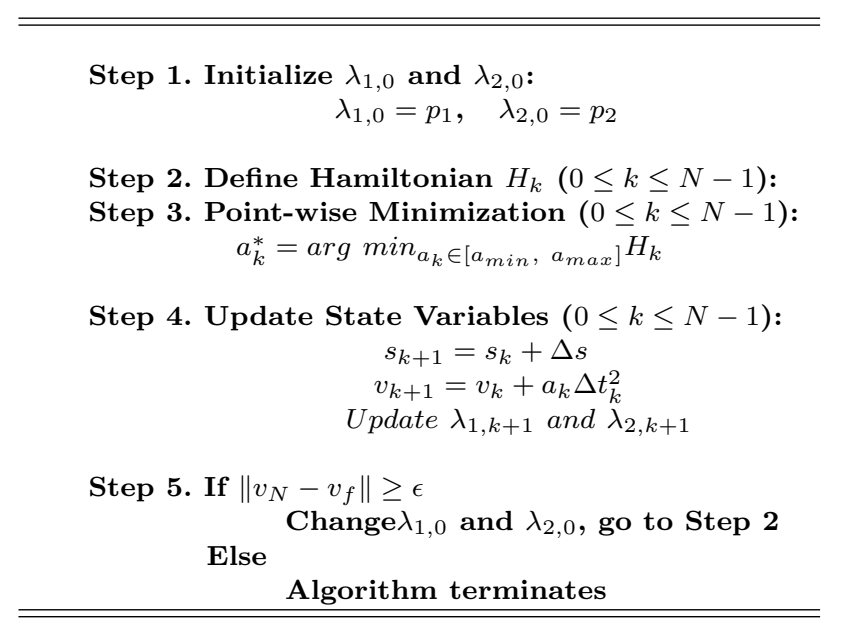

Table 2. Optimal Speed Planning Algorithm

$$
\delta_{i}=\left\{\begin{array}{cc}
1 & \text { if } E_{\text {eng, } i}>0 \\
0 & \text { otherwise }
\end{array}\right.
$$

\subsection{Fast Speed Planning Algorithm}

In order to solve the optimization problem (10), a Hamiltonian function for optimal control is introduced:

$$
\begin{aligned}
H_{k}\left(v_{k}, a_{k}, \Delta t_{k}, \lambda_{1, k}, \lambda_{2, k}\right) & =r\left(v_{k}, a_{k}\right) \Delta t_{k} \\
& +\lambda_{1, k} s_{k+1}+\lambda_{2, k} v_{k+1}
\end{aligned}
$$

where $\lambda_{1, k}$ and $\lambda_{2, k}$ are Lagrange multipliers. To find the minimum of (19) we apply the first order necessary condition to update state variables $s_{k}$ and $v_{k}$ and adjoint variables $\lambda_{1, k}$ and $\lambda_{2, k}$. The optimal speed planning algorithm is described in Table 2 .

\subsection{Point-wise Minimization}

The general way of performing point-wise minimization in Step 3 of Table 2 is to treat:

$$
\min _{a_{k} \in\left[a_{\text {min }}, a_{\text {max }}\right]} H_{k}\left(v_{k}, a_{k}, \Delta t_{k}, \lambda_{1, k}, \lambda_{2, k}\right)
$$

as a continuous problem and apply various optimization algorithm. This paper proposes a different method for point-wise minimization. Instead of solving a continuous problem and focusing on finding optimal $a_{k}$, it attempts to discretize the velocity and search for optimal $v_{k+1}$.

Given initial and final velocity $v_{0}$ and $v_{f}$ of the leading vehicle, Let:

$$
v_{\min }=\min \left\{v_{0}, v_{f}\right\}, \quad v_{\max }=\max \left\{v_{0}, v_{f}\right\}
$$

It is reasonable to restrict the velocity trajectory of leading vehicle within the region $v=v_{\min }, v=v_{\max }$, namely $v_{\min } \leq v_{k} \leq v_{\max }$ for all $k$. On the one hand, fuel consumption in acceleration case is much greater than steady state. On the other hand, OSP is a sort of shooting algorithm, bounding the velocity trajectory ensures the algorithm will not shoot out of the range, that is $v_{\min } \leq$ $v_{N} \leq v_{\max }$. Discretization on velocity space gives:

$$
\mathbf{v}=\left(v_{\min }, v_{\min }+\Delta v, \ldots v_{\min }+(m-1) \Delta v, v_{\max }\right)
$$

Step 1. Assign $B_{1, \min }, B_{1, \max }, B_{2, \min }$ and $B_{2, \max }$ : $B_{1, \min } \leq \lambda_{1,0} \leq B_{1, \max }$$$
B_{2, \min } \leq \lambda_{2,0} \leq B_{2, \max }
$$

Step 2. Divide the rectangular region into $N$ subregion equally, use the center of each subregion as $\left(\lambda_{1,0}, \lambda_{2,0}\right)$ and perform the OSP, compute corresponding $v_{N}$ and total fuel consumption.

Step 3. Keep the subregions which have $v_{N}=v_{f}$ and discard the rest.

Step 4. Find the subregion $R^{*}$ with minimum fuel consumption.

Step 5. Replace $B_{1, \min }, B_{1, \max }, B_{2, \min }, B_{2, \max }$ with coordinates of corners of the subregion, if $\sqrt{\left(B_{1, \max }-B_{1, \min }\right)^{2}+\left(B_{2, \max }-B_{2, \min }\right)^{2}}<\epsilon$ algorithm terminates, otherwise go back to Step 2.

Table 3. Algorithm for Initial Guess

In the next step one just simply applies the discrete optimization method to find the optimal $v_{k+1}^{*} \in \mathbf{v}$. The optimal $a_{k}$ can be calculated from $v_{k}$ and $v_{k+1}^{*}$ :

$$
a_{k}^{*}=\left\{\begin{array}{cc}
0 & \text { if } v_{k}=v_{k+1}^{*} \\
\frac{\left(v_{k+1}^{*}\right)^{2}-v_{k}^{2}}{2 \Delta s} & \text { otherwise }
\end{array}\right.
$$

\subsection{Initial Guess $\left(\lambda_{1,0}, \lambda_{2,0}\right)$}

It is known that OSP is a shooting algorithm and normally different initial guesses $\left(\lambda_{1,0}, \lambda_{2,0}\right)$ lead to various vehicle trajectories. Table 3 shows the general steps of finding $\left(\lambda_{1,0}, \lambda_{2,0}\right)$.

For example, let $N=10, B_{1, \min }=B_{2, \min }=-1000$ and $B_{1, \max }=B_{2, \max }=1000$, this means there are 100 regions and 100 associated centers of each region. We use the coordinate of center $\left(X_{i}, Y_{i}\right)$ as $\left(\lambda_{1,0}, \lambda_{2,0}\right)$ :

$$
\begin{aligned}
\lambda_{1,0} & =X_{i} \\
\lambda_{2,0} & =Y_{i}
\end{aligned}
$$

for the optimal speed planning algorithm. If $v_{N}=v_{f}$, we keep corresponding $\left(X_{i}, Y_{i}\right)$.

If there is only one $\left(X_{i}, Y_{i}\right)$ which gives $v_{N}=v_{f}$, then $\left(X_{i}, Y_{i}\right)$ is the solution. Otherwise, if there are at least two point, $\left(X_{1}, Y_{1}\right)$ and $\left(X_{2}, Y_{2}\right)$ such that the associated final state $v_{N}^{(1)}=v_{f}$ and $v_{N}^{(2)}=v_{f}$, then we compare the total energy consumption $Q_{1}$ and $Q_{2}$.

$$
\begin{array}{rr}
\text { If } Q_{1}<Q 2, & \text { keep }\left(X_{1}, Y_{1}\right) \\
\text { Otherwise, } & \text { keep }\left(X_{2}, Y_{2}\right)
\end{array}
$$

Assume that $\left(X_{1}, Y_{1}\right)$ is kept, since it is the center of the region, we replace $B_{1, \min }, B_{2, \min }, B_{1, \max }$ and $B_{2, \max }$ with the corners and repeat the same operations until $\sqrt{\left(B_{1, \max }-B_{1, \min }\right)^{2}+\left(B_{2, \max }-B_{2, \min }\right)^{2}}<\epsilon$. 
Table 4. Vehicle Parameters for Simulation

\begin{tabular}{|c|c|c|c|}
\hline Parameter & Symbol & Value & Unit \\
\hline Vehicle Mass & $M$ & 40000 & $\mathrm{~kg}$ \\
\hline Standard Gravity & $g$ & 9.8 & $\mathrm{~m} / \mathrm{s}^{2}$ \\
\hline Rolling Resistance Coefficient & $\mu$ & $7 \times 10^{-3}$ & - \\
\hline Air Drag Coefficient & $c_{D}$ & 0.6 & - \\
\hline Front Area & $A_{a}$ & 10.26 & $\mathrm{~m}^{2}$ \\
\hline Air Density & $\rho_{a}$ & 1.29 & $\mathrm{~kg} \bullet \mathrm{m}^{-3}$ \\
\hline Reduction Factor & $\phi$ & - & - \\
\hline
\end{tabular}

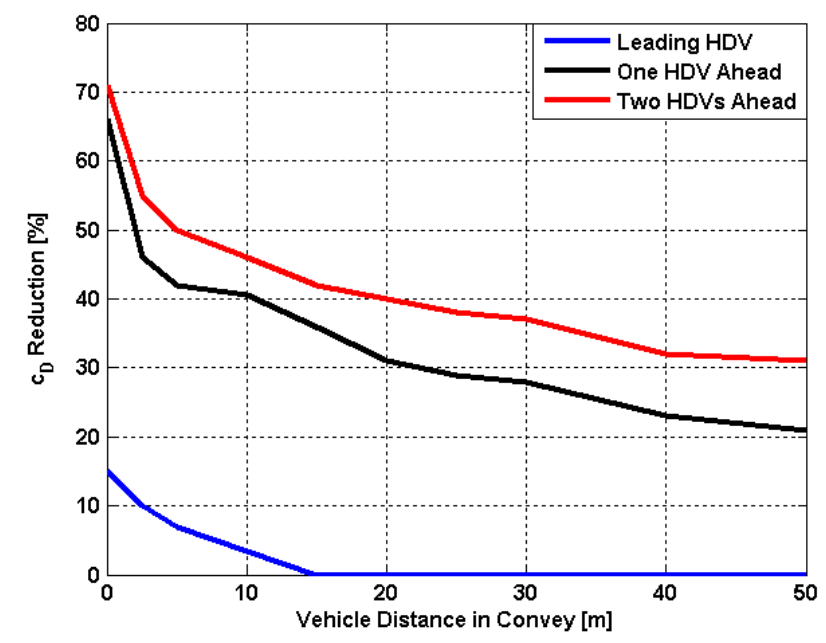

Fig. 1. Air-Drag Coefficient Reduction

Table 5. Energy Saving in Deceleration

\begin{tabular}{cccc}
\hline & $\begin{array}{c}\text { DP } \\
\text { Algorithm }\end{array}$ & $\begin{array}{c}\text { OSP } \\
\text { Algorithm }\end{array}$ & $\begin{array}{c}\text { Base Line } \\
\text { Driving }\end{array}$ \\
\cline { 2 - 4 } Energy $(J)$ & $5.3081 \times 10^{5}$ & $5.3477 \times 10^{5}$ & $7.6207 \times 10^{5}$ \\
Time $(s)$ & 156 & 7.7 & - \\
Energy Saving $(s)$ & $30.3 \%$ & $29.8 \%$ & - \\
\hline
\end{tabular}

\section{NUMERICAL EXAMPLE AND ANALYSIS}

In this paper we use the parameters in Table 4 ( [Lima 2013]) for simulation and estimate the reduction factor $\phi$ from Figure 1.

This section compares the solutions between One-Vehicle Dynamic Programming and One-Vehicle Optimal Speed Planning with Baseline (BL) Driving on energy consumption, algorithm running time and energy saving, and then illustrates the application of Optimal Speed Planning algorithm on a two-vehicle platooning with two main scenarios - deceleration and acceleration, assume that the travel distance $L=500$, the initial position of platoon leader $s_{0}=0$ :

\subsection{One $H D V$}

Deceleration Scenario: the vehicle starts with $v_{0}=25$ and decelerates to $v_{f}=5$ when it reaches $L$.

Acceleration Scenario: the vehicle starts with $v_{0}=5$ and accelerates to $v_{f}=25$ when it reaches $L$.

According to Table 5 and Table 6, although energy consumption in Optimal Speed Planning algorithm is a bit higher $(0.7 \%$ in deceleration scenario and $0.4 \%$ in acceler-
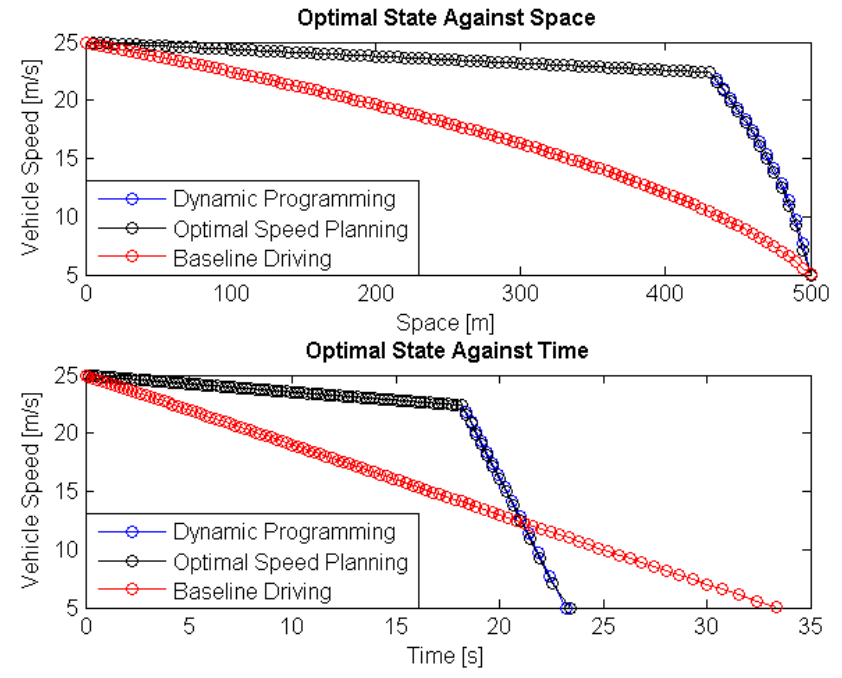

Fig. 2. One-Vehicle DP vs. OSP in Deceleration
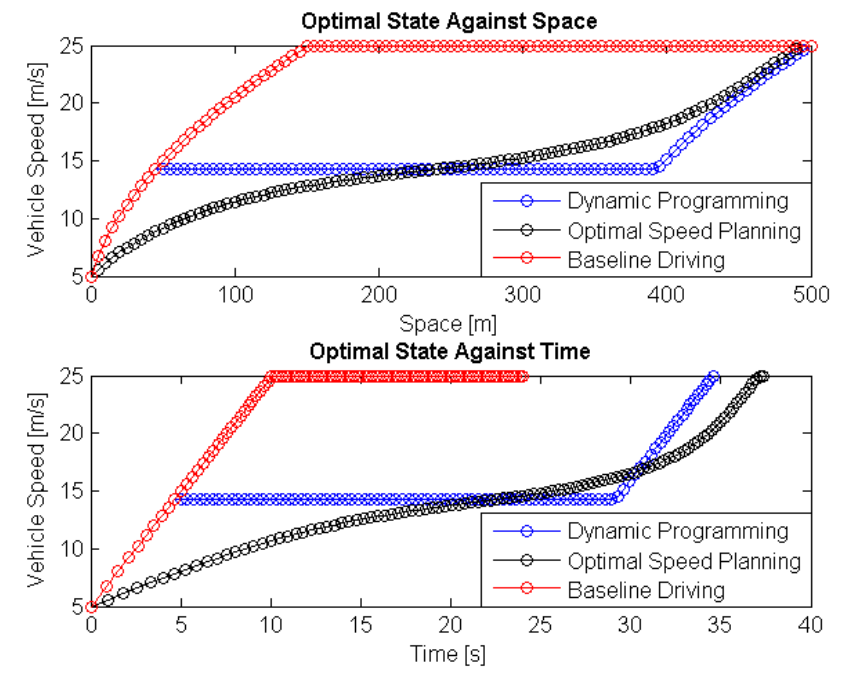

Fig. 3. One-Vehicle DP vs. OSP in Acceleration

Table 6. Energy Saving in Acceleration

\begin{tabular}{cccc}
\hline & $\begin{array}{c}\text { DP } \\
\text { Algorithm }\end{array}$ & $\begin{array}{c}\text { OSP } \\
\text { Algorithm }\end{array}$ & $\begin{array}{c}\text { Base Line } \\
\text { Driving }\end{array}$ \\
\cline { 2 - 4 } Energy $(J)$ & $1.4639 \times 10^{7}$ & $1.4702 \times 10^{7}$ & $1.4982 \times 10^{7}$ \\
Time $(s)$ & 141 & 7.8 & - \\
Energy Saving & $2.2 \%$ & $1.9 \%$ & - \\
\hline
\end{tabular}

ation) than DP algorithm, it is $94 \%$ - 95\% er than DP and thus more preferable.

In order to see the energy saving, we compare the optimal speed planning with baseline (BL) driving behavior in deceleration scenario, the driver tries to decelerate the HDV from highest speed $(25 \mathrm{~m} / \mathrm{s})$ to final speed $(5 \mathrm{~m} / \mathrm{s})$ with constant deceleration $a=0.5 *\left(25^{2}-5^{2}\right) / 500=$ $0.6 \mathrm{~m} / \mathrm{s}^{-2}$. DP saves $30.3 \%$ energy while Optimal Speed Planning saves $29.8 \%$.

We also compare the optimal speed planning with baseline (BL) driving behavior in acceleration scenario, the driver tries to accelerate with maximum acceleration $2 \mathrm{~m} / \mathrm{s}^{-2}$ to desired speed $(25 \mathrm{~m} / \mathrm{s})$, then keeps the HDV speed constant. DP and Optimal Speed Planning save $2.2 \%$ and $1.9 \%$ energy respectively. 
Table 7. Energy Consumption and Saving of Different Drivings in Deceleration

\begin{tabular}{cccc}
\hline $\begin{array}{c}\text { Energy } \\
(J)\end{array}$ & $\begin{array}{c}\text { BL Driving } \\
\text { No Platoon }\end{array}$ & $\begin{array}{c}\text { BL Driving } \\
\text { with Platoon }\end{array}$ & $\begin{array}{c}\text { Platoon } \\
\text { with OSP }\end{array}$ \\
\hline Leader & $7.6207 \times 10^{5}$ & $7.6207 \times 10^{5}$ & $5.3477 \times 10^{5}$ \\
Follower & $7.6207 \times 10^{5}$ & $7.6207 \times 10^{5}$ & $5.3477 \times 10^{5}$ \\
Total & $15.2414 \times 10^{5}$ & $15.2414 \times 10^{7}$ & $10.6954 \times 10^{5}$ \\
Saving & - & - & $29.8 \%$ \\
\hline
\end{tabular}

Table 8. Energy Consumption and Saving of Different Drivings in Acceleration

\begin{tabular}{cccc}
\hline $\begin{array}{c}\text { Energy } \\
(J)\end{array}$ & $\begin{array}{c}\text { BL Driving } \\
\text { No Platoon }\end{array}$ & $\begin{array}{c}\text { BL Driving } \\
\text { with Platoon }\end{array}$ & $\begin{array}{c}\text { Platoon } \\
\text { with OSP }\end{array}$ \\
\hline Leader & $1.4982 \times 10^{7}$ & $1.4979 \times 10^{7}$ & $1.4676 \times 10^{7}$ \\
Follower & $1.4982 \times 10^{7}$ & $1.4555 \times 10^{7}$ & $1.4245 \times 10^{7}$ \\
Total & $2.9964 \times 10^{7}$ & $2.9534 \times 10^{7}$ & $2.8921 \times 10^{7}$ \\
Saving & - & $1.4 \%$ & $3.5 \%$ \\
\hline
\end{tabular}

\subsection{Two-HDV Platoon with $A C C$}

In this subsection, we compare the energy consumption and saving of different drivings in deceleration and acceleration scenarios respectively.

Table 7 shows that the energy saving in Platoon with Optimal Speed Planning can be $29.8 \%$, yet no saving for just Baseline Driving with Platoon in the deceleration scenario, that because kinetic energy $\left(\frac{1}{2} M v^{2}\right)$ is more than sufficient for air-drag resistance and rolling resistance, namely, the vehicles do not spend any fuel to decrease the speed from $25 \mathrm{~m} / \mathrm{s}$ to $5 \mathrm{~m} / \mathrm{s}$, energy consumption comes from the Base Consumption such as air conditioner and other accessories.

However in acceleration scenario, according to Table 8, the energy reduction in Normal Driving with Platoon is $1.4 \%$ and it could be further enhanced to $3.5 \%$ when Optimal Speed Planning is applied $(2.1 \%$ for the platoon leader and $5 \%$ for the follower) due to the fact that the reduction of air-drag resistance can also be enhanced by Optimal Speed Planning.

\section{CONCLUSION AND PERSPECTIVES}

This paper proposes a fast algorithm for planning platoon speed in the context of real-time fleet management application. The main advantage of the algorithm is its computation efficiency in comparison to the previous approach using dynamic programming. This makes it a better candidate for real system when local speed of a platoon has to be continuously adapted (controlled) according to real time traffic information. The method is considered useful for scenarios that truck platoon needs to carry out acceleration, deceleration or integrated operations. Indeed, it is also applicable for strategic planning of platoon speed when traffic states on links of a planned route are generally available.

While the methodology is towards the general platooning problem, the numerical study focus mainly on a platoon of two trucks during the scenarios in acceleration and deceleration. The method can be easily extended for longer platoon if needed. Numerical experiments show that significant energy saving during deceleration scenario $(29.8 \%)$ and acceleration scenario (3.5\%) using the the parameters
$N=100, \Delta v=0.01$. The short calculation time, shown in Table 5 and 6 , prove the significant improvement on computational time in comparison to the DP based solution algorithm. On the other hand, there is only small difference between the results from two approaches, with maximum $0.5 \%$ in our studies. This indicates that the proposed approach is a preferred solution, although it is a suboptimal solution. One limitation of the study is that the approach in choosing initial $\lambda_{1,0}$ and $\lambda_{2,0}$ is still not so efficient. This has to be addressed in the future by further exploration on how $\Delta s$ and $\Delta v$ affect the algorithm.

\section{ACKNOWLEDGEMENTS}

The authors would like to acknowledge the financial support of the iQFleet project by the Swedish Innovation Agency (Vinnova). The participation of the IFAC congress is partially supported by the Swedish ITS Postgraduate School.

\section{REFERENCES}

Browand, F., McArthur, J., and Radovich, C. (2004). Fuel saving achieved in the field test of two tandem trucks. Technical Report UCB-ITS-PRR-2004-20, California PATH research report.

Donikian, S., Espie, S., Parent, M., and Rousseau, G. (1998). Simulation studies on the impact of acc. In proceedings of the 5th World Congress on Intelligent Transport Systems.

Lima, P. (2013). Implementation and Analysis of Platoon Catch-Up Scenarios for Heavy Duty Vehicles. Master's thesis, KTH Electrical Engineering.

Franke, U., Böttiger, F., Zomotor, Z., and Seeberger, D. (1995). Truck platooning in mixed traffic. In Proceedings of the IEEE Intelligent Vehicle Symposium.

Godbole, D. and Lygeros, J. (1993). Longitudinal Control of the Lead Car of a Platoon, volume 93-7.

Hooker, J. (1988). Optimal driving for single-vehicle fuel economy. Transportation Research A, 22A(3), 183-201.

Ma, X. (2013). Towards intelligent fleet management: Local optimal speeds for fuel and emissions. In Proceedings of the 16th IEEE international conference on Intelligent Transportation Systems.

Oguchi, T., Katakura, M., and Taniguchi, M. (2002). Carbon dioxide emission model in actual urban road vehicular traffic conditions. Infrastructure Planning and Management, No. 695/IV-54, pp. 125-136, January 2002.

Shladover, S., Desoer, C., Hedrick, J., Tomizuka, M., Walrand, J., Zhang, W.B., McMahon, D., Peng, H., and S. Sheikholeslam McKeown, N. (1991). Automatic vehicle control development in the PATH program. IEEE Trans. on Vechiular Technology, 40:1, 114-130.

Stankovic, S., Stanojevic, M., and Siljak, D. (2013). Stochastic decentralized control of a platoon of vehicles based on the inclusion principle. Proceedings in Mathematics \&f Statistics, 31, 223-238. 